\title{
Article \\ Genome-Wide Identification of the $B-B O X$ Genes that Respond to Multiple Ripening Related Signals in Sweet Cherry Fruit
}

\author{
Yanyan Wang, Zefeng Zhai, Yueting Sun, Chen Feng, Xiang Peng, Xiang Zhang, Yuqin Xiao, Xin Zhou, Weili Wang, \\ Jiale Jiao and Tianhong $\mathrm{Li}$ *
}

Citation: Wang, Y.; Zhai, Z.; Sun, Y.; Feng, C.; Peng, X.; Zhang, X.; Xiao, Y.; Zhou, X.; Wang, W.; Jiao, J.; et al. Genome-Wide Identification of the B-BOX Genes that Respond to Multiple Ripening Related Signals in Sweet Cherry Fruit. Int. J. Mol. Sci. 2021, 22, 1622. https://doi.org/ $10.3390 /$ ijms 22041622

Academic Editors: Soulaiman Sakr Sadanad A. Dhekney and Jayasankar Subramanian Received: 5 January 2021

Accepted: 3 February 2021

Published: 5 February 202

Publisher's Note: MDPI stays neutra with regard to jurisdictional claims in published maps and institutional affiliations.

Copyright: (c) 2021 by the authors. Licensee MDPI, Basel, Switzerland. This article is an open access article distributed under the terms and conditions of the Creative Commons Attribution (CC BY) license (https:// creativecommons.org/licenses/by/ $4.0 /)$
Department of Pomology, College of Horticulture, China Agricultural University, Beijing 100193, China; yabofei1212@163.com (Y.W.); zhaizefeng@126.com (Z.Z.); yuetingsun@126.com (Y.S.); fengc@cau.edu.cn (C.F.); rod@cau.edu.cn (X.P.); 18306391375@163.com (X.Z.); S20193172433@cau.edu.cn (Y.X.); zx51522zzwlwlbb@126.com (X.Z.); WWL0824@foxmail.com (W.W.); qingxuedanchen@163.com (J.J.)

* Correspondence: lith@cau.edu.cn; Tel.: +86-010-6273-3957

\begin{abstract}
B$ - $B O X$ proteins are zinc finger transcription factors that play important roles in plant growth, development, and abiotic stress responses. In this study, we identified 15 PavBBX genes in the genome database of sweet cherry. We systematically analyzed the gene structures, clustering characteristics, and expression patterns of these genes during fruit development and in response to light and various hormones. The PavBBX genes were divided into five subgroups. The promoter regions of the PavBBX genes contain cis-acting elements related to plant development, hormones, and stress. qRT-PCR revealed five upregulated and eight downregulated PavBBX genes during fruit development. In addition, PavBBX6, PavBBX9, and PavBBX11 were upregulated in response to light induction. We also found that $\mathrm{ABA}, \mathrm{BR}$, and $\mathrm{GA}_{3}$ contents significantly increased in response to light induction. Furthermore, the expression of several PavBBX genes was highly correlated with the expression of anthocyanin biosynthesis genes, light-responsive genes, and genes that function in multiple hormone signaling pathways. Some PavBBX genes were strongly induced by ABA, GA, and BR treatment. Notably, PavBBX6 and PavBBX9 responded to all three hormones. Taken together, $B B X$ proteins likely play major roles in regulating anthocyanin biosynthesis in sweet cherry fruit by integrating light, ABA, GA, and BR signaling pathways.
\end{abstract}

Keywords: Prunus avium; B-box; fruit ripening; light; hormones

\section{Introduction}

Zinc finger proteins are transcription factors that play crucial roles in plants [1], including functions related to development and stress resistance [2]. BBX family zinc finger transcription factors have attracted increasing attention due to their multiple, important roles in plants $[3,4]$. For example, the $B B X$ domains and CCT domains of $B B X$ proteins play specific roles in transcriptional regulation and in protein interactions and nuclear transport, respectively [5,6]. Many studies have demonstrated that $B B X$ proteins are essential for photomorphogenesis, floral induction, carotenoid biosynthesis, the shade avoidance response, and both biotic and abiotic stress resistance [3,7].

To date, 32 BBX proteins have been identified in Arabidopsis thaliana. These proteins were classified into five groups based on their domain characteristics [5] and are involved in regulating many biological processes, including flowering and photomorphogenesis [8-10]. $\mathrm{CO} / A t B B X 1$ plays a central role in the photoperiodic regulation of flowering in Arabidopsis, and COL3/AtBBX4 and COL9/AtBBX7 participate in the regulation of flowering time [11,12]. Photomorphogenesis is positively regulated by AtBBX11 [13], AtBBX21 [3], $A t B B X 22$ [14], and AtBBX23 [15] in Arabidopsis. By contrast, photomorphogenesis is negatively regulated by $A t B B X 24$ [16,17], AtBBX25 [18], AtBBX28/29 [19], AtBBX30/31 [20]. In Arabidopsis. HY5 is the core component of photomorphogenesis [21]. AtBBX21 physically interact with $H Y 5$, and binds to the T/G-box cis-element in the promoter of $H Y 5$ to activate 
its transcription [3]. Interesting, $H Y 5$ can bind to the promoter region of $B B X 11, B B X 11$ can also directly bind to the promoter region of $H Y 5$ and positively regulate its expression. $B B X 11-B B X 21-H Y 5$ form a positive feedback regulation system at the transcription level, which cooperatively promotes photomorphogenesis [13]. On the other hand, HY5 binds to the promoters of $B B X 30$ and $B B X 31$ and inhibits their activity [22,23]. In addition, $B B X 28$ and $B B X 29$ negatively regulates photomorphogenesis by repressing the activity of transcription factor $H Y 5$, furthermore, both $B B X 28$ and $B B X 29$ interfere with the binding of $H Y 5$ to the promoters of $B B X 30$ and $B B X 31[19,24,25]$. BBX28/BBX29, HY5, and $B B X 30 / 31$ form a feedback loop to fine-tune photomorphogenic development [25]. Therefore, $B B X s$ form a complex regulatory network with $H Y 5$ to regulate photomorphogenesis [24].

$B B X$ genes are also indispensable for abiotic stress responses and hormone signaling. $A t B B X 24$ is involved in salt stress signaling, as Arabidopsis plants overexpressing AtBBX24 exhibited enhanced salt tolerance compared to wild-type plants [26]. In apple (Malus domestica), MdBBX20 mediates UV-B and low temperature signaling to promote anthocyanin biosynthesis [27], and MdBBX37 interacts with MdMYB1 and MdMYB9 to negatively regulate anthocyanin biosynthesis [28]. $P p B B X 16$ positively regulates light-induced anthocyanin accumulation in Asian pear (Pyrus pyrifolia) by activating PpMYB10 expression [29]. $C m B B X 19$ interacts with $\mathrm{ABF} 3$ to negatively regulate drought tolerance in chrysanthemum (Chrysanthemum morifolium) [30]. In Arabidopsis, COL4/AtBBX5 participates in salt stress responses through an ABA-dependent signaling pathway [31]. In addition, AtBBX18 is involved in the gibberellic acid (GA) signal transduction pathway, and $A t B B X 20$ is involved in the brassinolide (BR) and light signal pathways [32,33]. IbBBX24 improves the resistance of sweet potato (Ipomoea batatas) to Fusarium wilt via the jasmonic acid (JA) pathway [34].

Sweet cherry (Prunus avium L.), a fruit tree in the Rosaceae family, is one of the deciduous fruit trees [35]. Red-skinned sweet cherries are distinguished into dark-red and bicolored cultivars, and 'Rainier' is the one of the main bicolored cultivars recognized in the world, including in China. Our previous study demonstrated that light plays a major role in anthocyanin accumulation in bicolored but not dark-red cherries [36]. Therefore, in this study, 'Rainier' was used as the material to analyze the molecular mechanisms by which $B B X$ genes participate in the regulation of light- and hormone-induced anthocyanin accumulation in sweet cherry, we identified and investigated 15 PavBBX family members in the genome database of sweet cherry. We analyzed their gene structures, phylogenetic relationships, and expression profiles in response to light and multiple hormone signals. Our results lay the foundation for further analyzing the roles of $B B X$ genes sweet cherry fruit development and ripening.

\section{Results}

\subsection{Identification of PavBBX Genes in Prunus avium}

To identify the $B B X$ genes in the sweet cherry genome, we used Arabidopsis $B B X$ protein sequences as queries to search against the sweet cherry genome via BLAST. We verified the results of the BLAST search by confirming the presence of the B-box domain in each protein using SMART. After removing the redundant sequences, a total of 15 putative PavBBX genes were identified in sweet cherry, which were named PavBBX1-PavBBX15 based on their locations in the reference genome (Figure S1). Detailed information about the PavBBX genes is provided in Table 1, including the gene name, protein length, chromosome location, molecular weight, theoretical isoelectric point, aliphatic index, and GRAVY value. The 15 PavBBX proteins had diverse molecular weights and lengths, ranging from 152 (PavBBX6) to 650 (PavBBX1) amino acids long. PavBBX6 had the lowest molecular weight $(17.01 \mathrm{kDa})$, while PavBBX1 had the highest $(71.17 \mathrm{kDa})$. The theoretical isoelectric points of the PavBBX proteins ranged from 4.51 (PavBBX8) to 9.32 (PavBBX4), and the aliphatic indices ranged from 38.68 (PavBBX8) to 82.97 (PavBBX15). The GRAVY values of all PavBBXs were less than zero, pointing to the hydrophilic nature of PavBBX proteins (Table 1). 
Table 1. BBX family genes in Prunus avium.

\begin{tabular}{|c|c|c|c|c|c|c|c|c|c|}
\hline $\begin{array}{l}\text { Gene } \\
\text { Name }\end{array}$ & Accession Number & Protein/AA & Chrom & Chr Start & Chr End & $\begin{array}{l}\text { MW } \\
\text { (Da) }\end{array}$ & pI & $\begin{array}{l}\text { Aliphatic } \\
\text { Index }\end{array}$ & GRAVY \\
\hline PavBBX1 & Pav_sc0004527.1g080.1 & 650 & Chr1 & 17737787 & 17743220 & $71,169.18$ & 5.68 & 74.68 & -0.458 \\
\hline PavBBX2 & Pav_sc0001051.1g030.1 & 450 & Chr1 & 24564167 & 24566102 & $49,926.26$ & 5.15 & 56.84 & -0.790 \\
\hline PavBBX3 & Pav_sc0000131.1g850.1 & 343 & Chr1 & 29989611 & 29991028 & $38,307.77$ & 5.47 & 65.95 & -0.539 \\
\hline$P a v B B X 4$ & Pav_sc0000293.1g410.1 & 264 & Chr3 & 661262 & 662056 & $28,357.08$ & 9.32 & 72.16 & -0.246 \\
\hline PavBBX5 & Pav_sc0000405.1g160.1 & 399 & Chr3 & 16146055 & 16148421 & $42,967.79$ & 5.07 & 61.13 & -0.485 \\
\hline PavBBX6 & Pav_sc0001859.1g200.1 & 152 & Chr3 & 16934620 & 16935833 & $17,011.06$ & 9.09 & 60.46 & -0.849 \\
\hline PavBBX7 & Pav_sc0000051.1g110.1 & 324 & Chr3 & 18112949 & 18115389 & $35,601.87$ & 5.78 & 67.25 & -0.482 \\
\hline PavBBX8 & Pav_sc0000598.1g150.1 & 204 & Chr4 & 1931059 & 1932064 & $23,047.58$ & 4.51 & 38.68 & -1.254 \\
\hline PavBBX9 & Pav_sc0000352.1g070.1 & 301 & Chr4 & 11752866 & 11755126 & $31,786.49$ & 5.53 & 62.86 & -0.317 \\
\hline PavBBX10 & Pav_sc0000383.1g410.1 & 480 & Chr5 & 15713586 & 15715861 & $53,565.68$ & 5.12 & 62.23 & -0.770 \\
\hline PavBBX11 & Pav_sc0000044.1g810.1 & 229 & Chr7 & 13871819 & 13874309 & $25,370.08$ & 8.51 & 74.10 & -0.387 \\
\hline PavBBX12 & Pav_sc0001518.1g660.1 & 208 & Chr8 & 8383526 & 8384850 & $23,011.66$ & 5.97 & 58.17 & -0.462 \\
\hline PavBBX13 & Pav_sc0000848.1g470.1 & 391 & Chr8 & 19897720 & 19900725 & $43,198.97$ & 6.16 & 58.90 & -0.661 \\
\hline PavBBX14 & Pav_sc0001100.1g020.1 & 239 & ChrUn & 27735116 & 27739262 & $26,178.43$ & 4.77 & 74.81 & -0.315 \\
\hline PavBBX15 & Pav_sc0002706.1g040.1 & 333 & ChrUn & 55031701 & 55033349 & $35,617.19$ & 4.95 & 82.97 & -0.046 \\
\hline
\end{tabular}

2.2. Protein Sequence Alignment and Phylogenetic Analysis of the PavBBX Gene Family

The lengths of the sweet cherry $B B X$ proteins varied widely, from 152 to 650 amino acids. Among these, four PavBBXs contained two B-box domains and a conserved CCT domain. Overall, six PavBBXs contained two B-box domains but no CCT domain; additionally, two PavBBXs contained one B-box domain and a CCT domain, and three contained only one $B$-box domain (Figure 1). Protein sequence alignment and WebLogo analysis revealed that the CCT domains in the proteins were conserved and that the two $B$-box domains were highly homologous in different $B B X$ proteins (Figures S2 and S3).

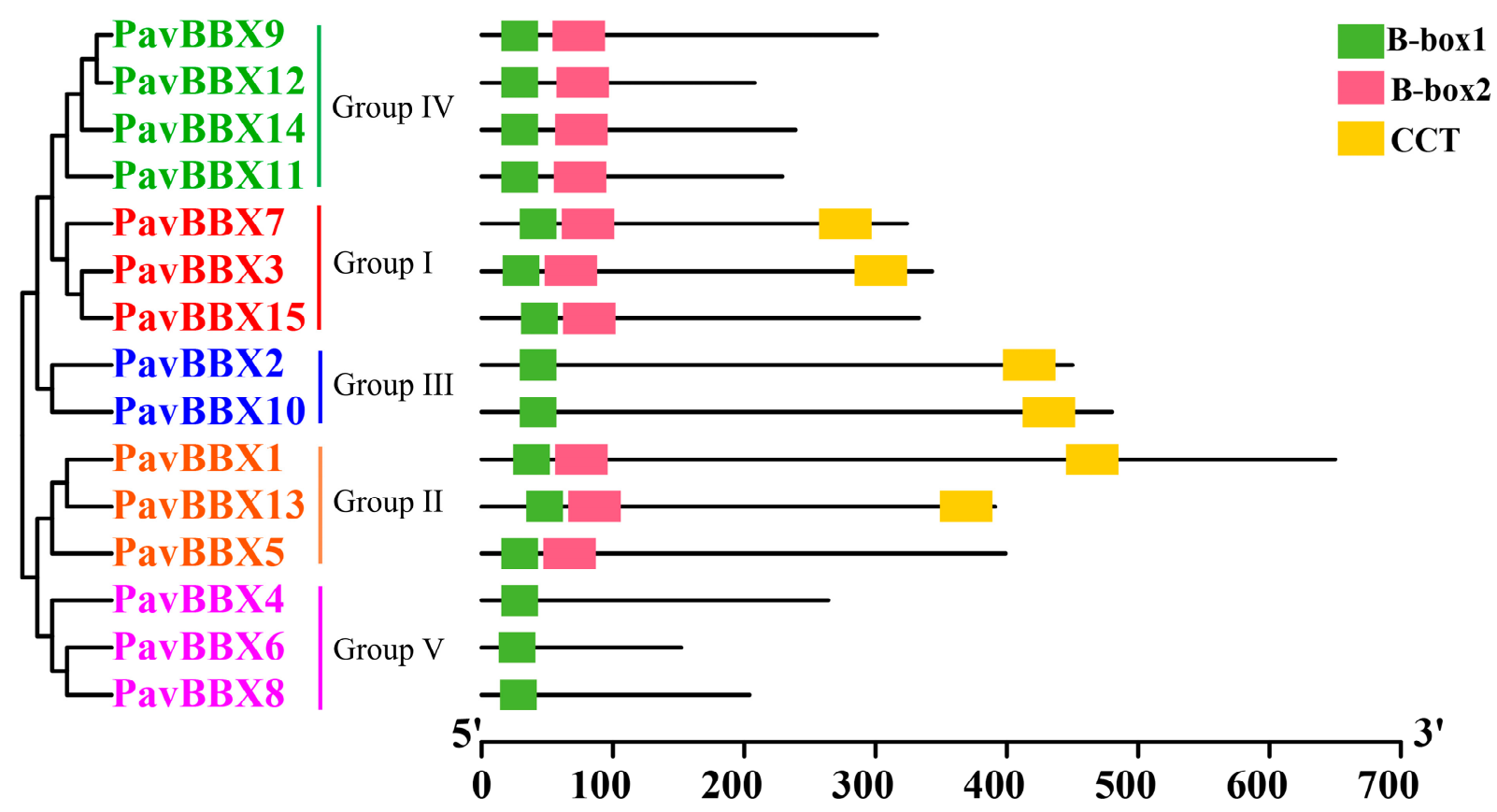

Figure 1. Structures of Prunus avium BBX proteins. The green, pink, and yellow rectangles represent the $B$-box1, B-box2, and CCT domains, respectively. The scale bar represents amino acids. 
To explore the evolutionary relationships and functional divergence of the PavBBX family members, we constructed a phylogenetic tree with MEGA6.0 using the neighborjoining method based on various $P a v B B X, A t B B X, P b B B X$, and $S l B B X$ protein sequences (Figure 2). The $B B X$ family was divided into five subgroups based on the results of phylogenetic analysis and previous studies in Arabidopsis [5], tomato [37] and pear [38]. PavBBX subgroup I and subgroup II members contain two $B$-box and one CCT domains, except for PavBBX5 and PavBBX15. Subgroup III members contain one B-box and one CCT domain. PavBBX subgroup IV and V members contain two or one $B$-box domain (s) and no CCT domains.

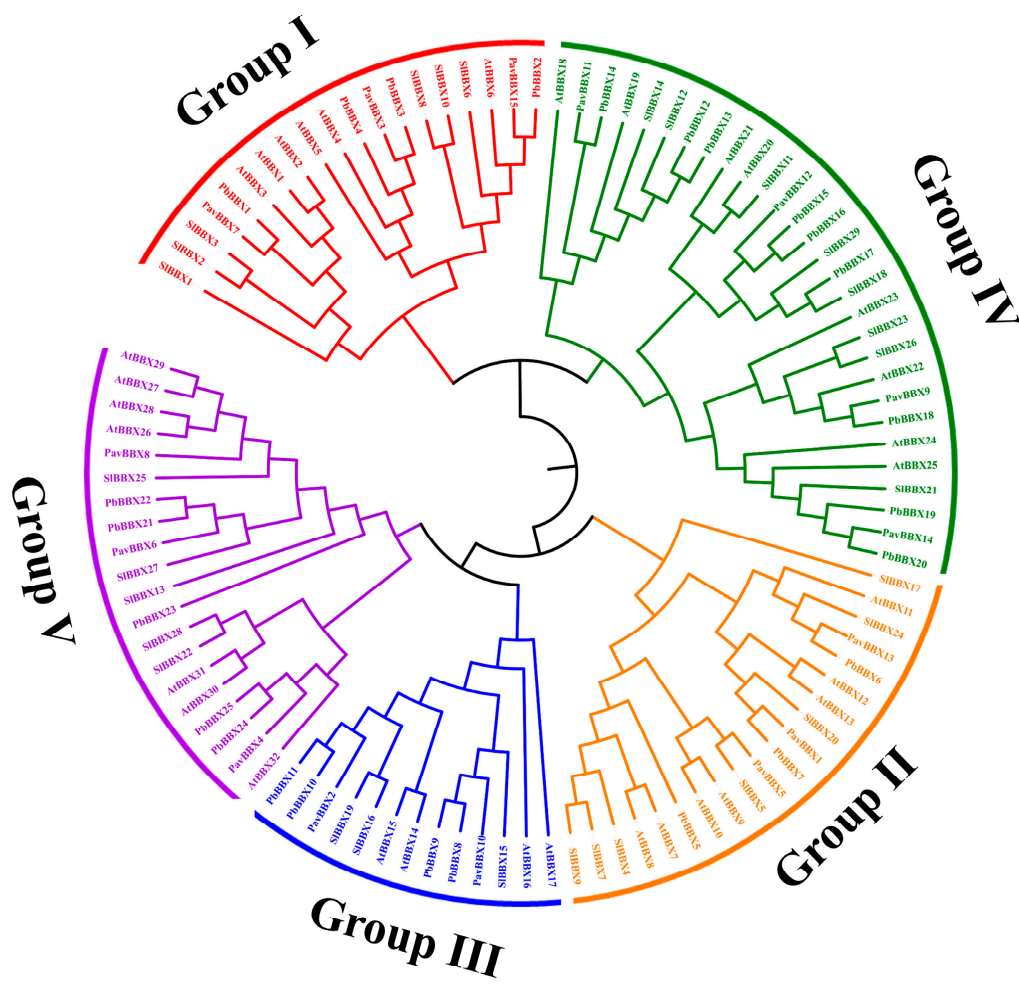

Figure 2. Phylogenetic analysis of BBX genes in sweet cherry (Prunus avium), pear (Pyrus bretschneideri Rehd), tomato (Solanum lycopersicum), and Arabidopsis (Arabidopsis thaliana).

\subsection{Cis-Elements in the Promoters of Sweet Cherry BBX Genes}

Cis-elements are involved in regulating gene expression by interacting with their corresponding trans-regulators. Identifying proposed cis-elements would provide valuable information about the expression of the sweet cherry $B B X$ genes. We therefore examined the promoter regions of the PavBBX genes and used the PlantCARE database for cis-element prediction (Figure 3). We identified 26 predicted cis-elements in these promoter regions. Among these, CAAT-box and TATA-box elements were present in all PavBBX genes. The remaining elements included light-responsive elements, defense response elements, and hormone-responsive elements, such as those induced by abscisic acid (ABA), auxin (IAA), gibberellin (GA), SA, and MeJA. These findings explained that PavBBX genes function in a variety of abiotic stress responses. 


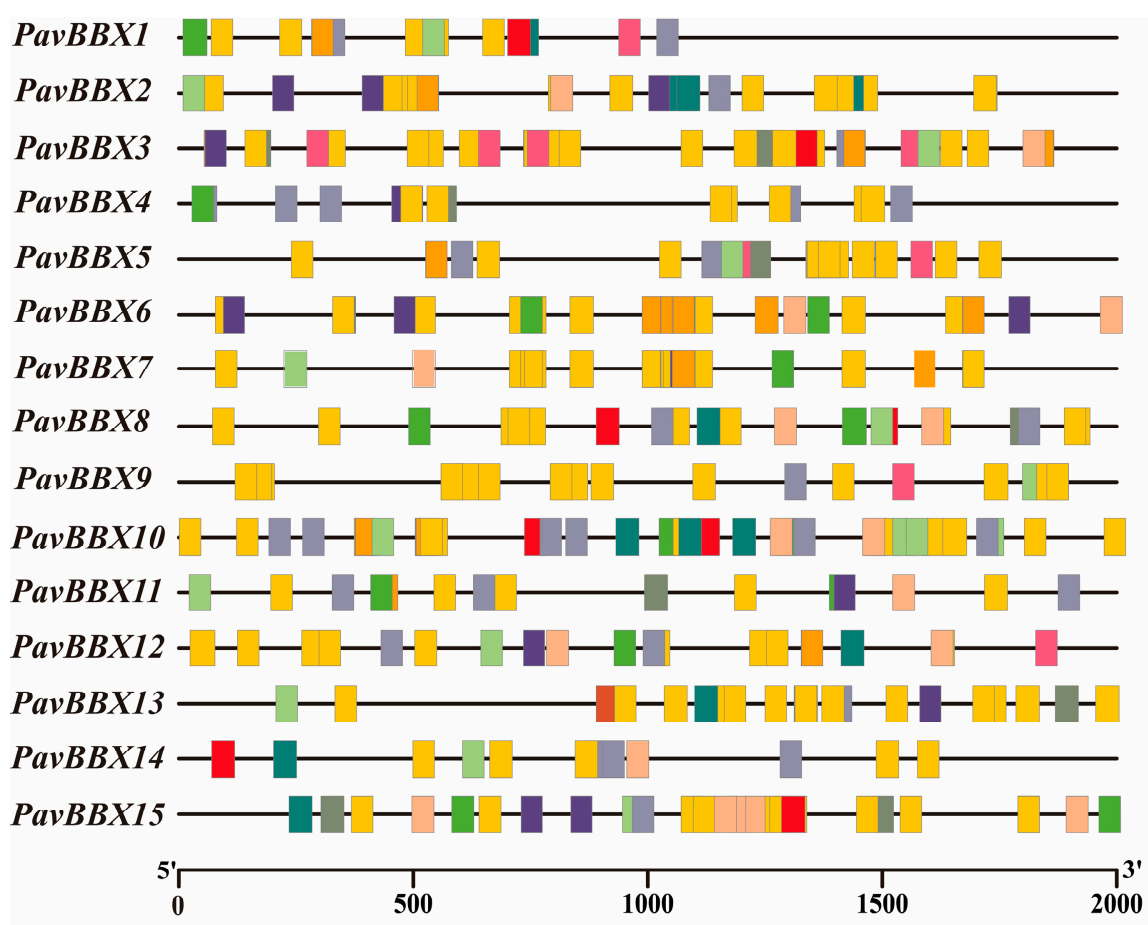

\begin{tabular}{|l|}
\hline Auxin response element \\
\hline Light response element \\
\hline Low temperature response element \\
\hline Zein metabolism regulation element \\
\hline SA response element \\
\hline Anaerobic-induced element \\
\hline Drought-induced element \\
\hline ABA response element \\
\hline MeJA response element \\
\hline Gibberellin response element \\
Defense and stress response element \\
Wound response element
\end{tabular}

Figure 3. Schematic representation of the predicted regulatory cis-elements in the promoters of PavBBX family genes. The scale bar represents base pair.

\subsection{Expression Patterns of PavBBX Genes during Sweet Cherry Fruit Development and Ripening}

To analyze the roles of PavBBX genes in fruit development and maturation, we examined the expression patterns of the 15 PavBBX genes in sweet cherry fruit during three developmental stages. Different PavBBX genes showed distinct expression patterns during different stages of fruit development. As shown in Figure 4, PavBBX4, PavBBX6, PavBBX7, PavBBX9, and PavBBX11 were upregulated during fruit development, eight PavBBX genes were downregulated (PavBBX1, 2, 3, 5, 10, 12, 13, and 15), and the expression of the two remaining genes did not significantly change during fruit development. These results speculated that some PavBBX genes play multiple, important roles in sweet cherry fruit development.

\subsection{Regulation of PavBBX Gene Expression during Light Induction}

$B B X$ genes are involved in photomorphogenesis in a variety of plants [14,39]. Anthocyanin accumulation is highly dependent on light in bicolored Rainier cherries [36]. Therefore, to investigate the roles of the PavBBX genes in the plant response to light induction in bicolored 'Rainier' cherries, we placed bags on developing cherries at 15 DAF to block their exposure to light and removed the bags at 45 DAF. The fruits rapidly changed color after bag removal (Figure 5a), and anthocyanin continuously accumulated at $24 \mathrm{~h}$ after bag removal until the end of the experiment ( $96 \mathrm{~h}$ after bag removal) (Figure $5 \mathrm{~b}$ ). We used qRT-PCR to measure PavBBX genes expression in sweet cherry after bag removal. Different genes showed different expression levels (Figure $5 c$ ). As shown in Figure $5 d$, $\operatorname{PavBBX} 3,4,6,7,9,11$, and 15 were upregulated more than two-fold after light induction. In particular, $P a v B B X 9$ was upregulated approximately 10 -fold at $12 \mathrm{~h}$ of light induction and PavBBX6 and PavBBX11 were upregulated 4-5-fold at $24 \mathrm{~h}$ and $96 \mathrm{~h}$ of light induction, respectively. These results represented that these $B B X$ genes function in light-induced anthocyanin biosynthesis in sweet cherry. 


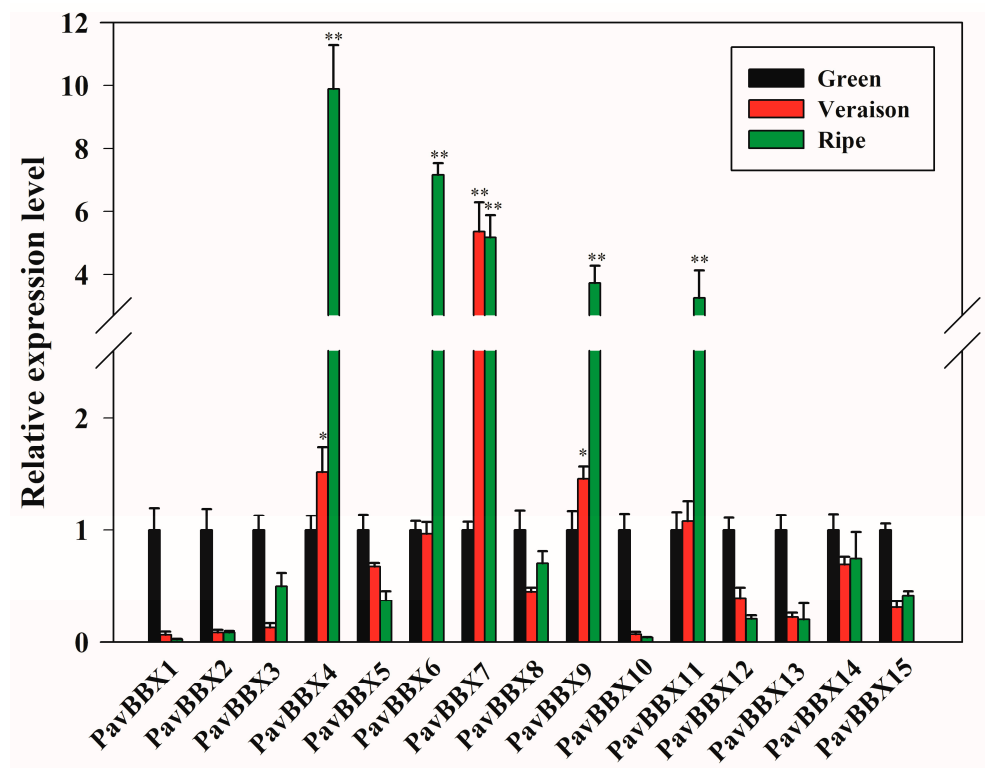

Figure 4. Expression profiles of the sweet cherry PavBBX genes during three stages of fruit development. PavActin was used as the internal reference control to normalize template levels. The relative mRNA levels are represented as the mean $\pm S D(n=3)$. Statistically significant differences were assessed using Student's $t$-test $\left.{ }^{*} p<0.05,{ }^{* *} p<0.01\right)$.

(a)

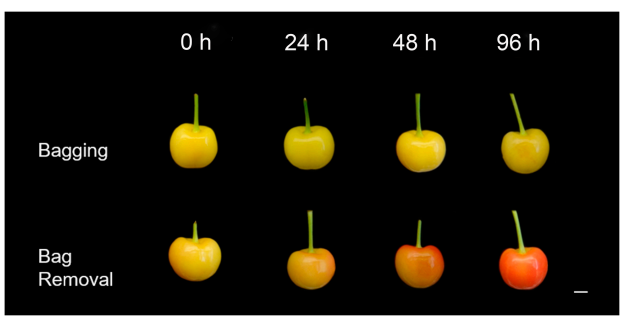

(b)

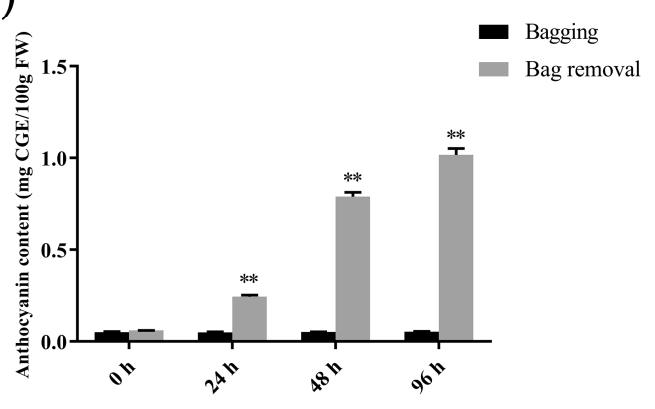

(c)

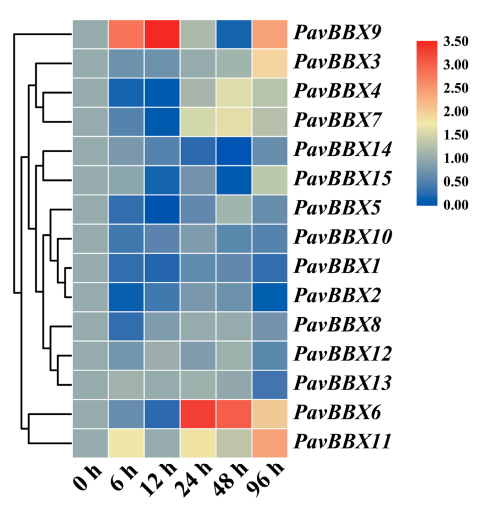

(d)

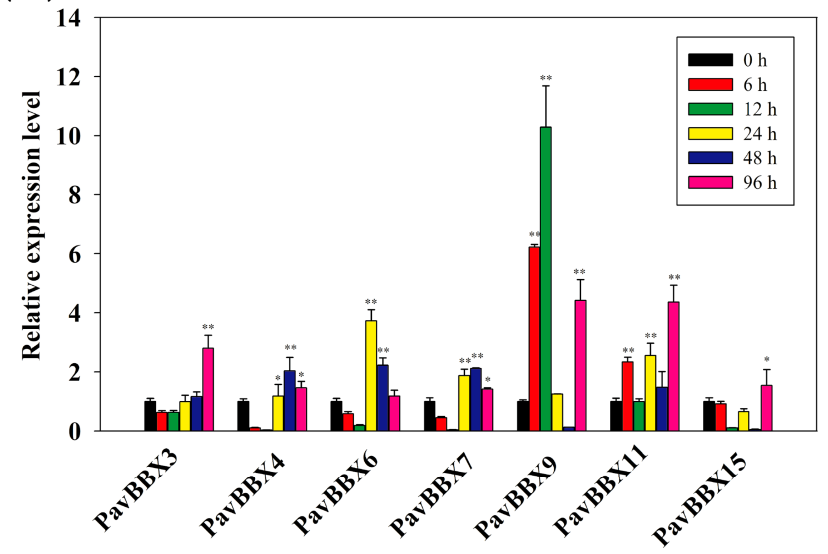

Figure 5. Expression profiles of PavBBX genes in 'Rainier' fruit following light induction via bag removal. (a) Color changes of 'Rainier' fruits after bag removal. Scale bars represent $1 \mathrm{~cm}$. (b) Changes in anthocyanin content. (c) Hierarchical clustering of the expression profiles of 15 PavBBX genes after bag removing. (d) RT-qPCR analysis of seven selected PavBBX genes following light induction. PavActin was used as the internal reference control to normalize template levels. The relative mRNA levels are represented as the mean $\pm \mathrm{SD}(\mathrm{n}=3)$. Statistically significant differences were assessed using Student's $t$-test $\left({ }^{*} p<0.05,{ }^{* *} p<0.01\right)$. 


\subsection{Light-Induced Regulation of Hormone Content in Sweet Cherry Fruit}

Plant hormones play important roles in inducing anthocyanin accumulation and commonly interact with light-signaling pathways to regulate plant growth [40]. Recent transcriptome expression studies have shown that anthocyanin biosynthesis in sweet cherry fruit involves plant hormone signaling, including ABA, auxin, BR, GA, and JA signaling pathways [36]. To determine whether light and plant hormones synergistically regulate anthocyanin accumulation in fruits, we measured hormone contents in 'Rainier' fruits after bag removal via ELISA. As shown in Figure 6, ABA contents significantly increased in fruits at 48 and $96 \mathrm{~h}$ after bag removal. In addition, $\mathrm{BR}$ and $\mathrm{GA}_{3}$ contents rapidly increased (2- to 3 -fold) at $12 \mathrm{~h}$ after bag removal. However, IAA and MeJA contents did not significantly differ in fruits before and after bag removal. These results indicated that light regulates anthocyanin accumulation, at least in part, by modulating $\mathrm{ABA}, \mathrm{GA}_{3}$, and $\mathrm{BR}$ signaling.
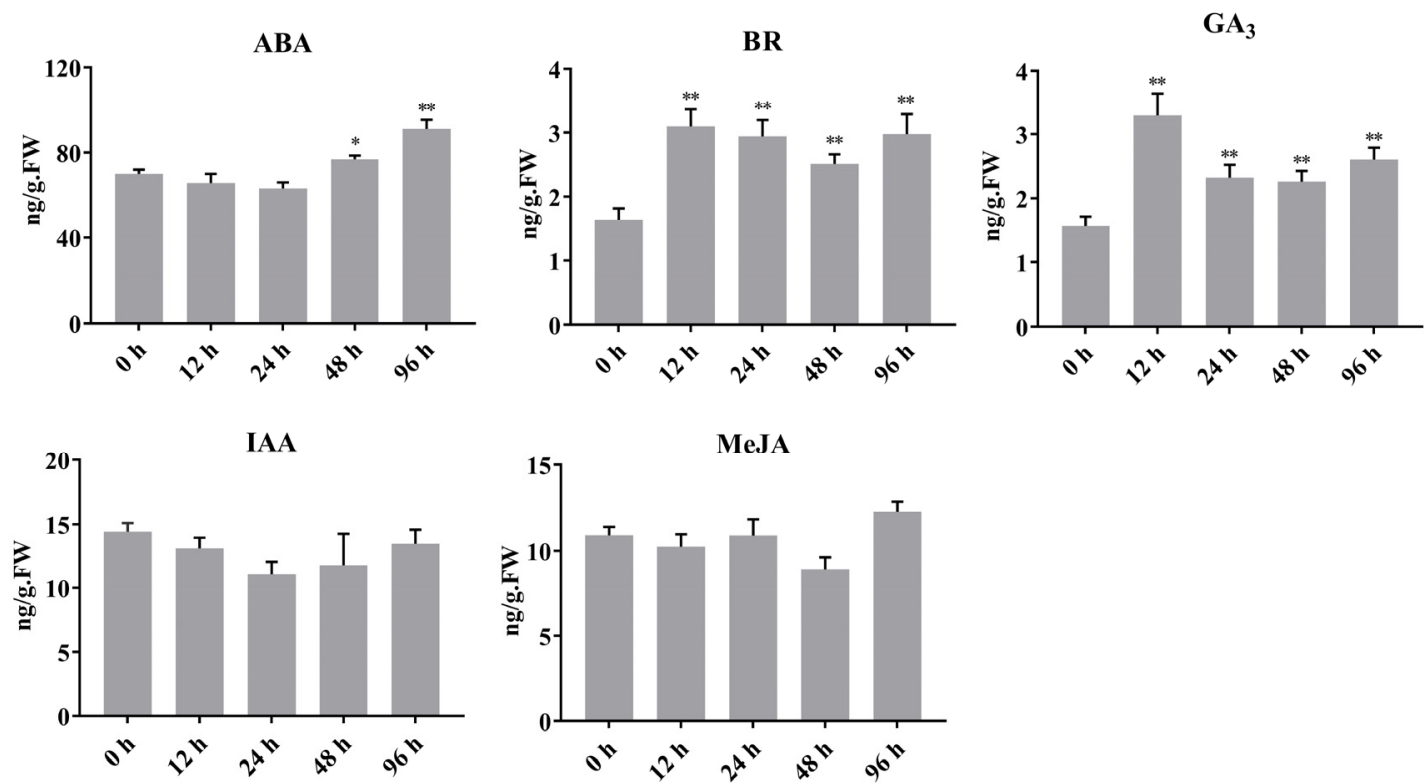

Figure 6. Changes in ABA, IAA, BR, MeJA, and GA 3 contents in 'Rainier' fruits after bag removal. Error bars on each column represent the standard error (S.E.) of three replicates. Statistically significant differences were assessed using Student's $t$-test $\left({ }^{*} p<0.05,{ }^{* *} p<0.01\right)$.

2.7. Co-Expression Network Analysis of PavBBX Genes with Anthocyanin Biosynthesis Genes, Light Signaling Genes, and Hormone Signaling Genes

We assessed the RNA-seq data (SAMN09296232 (RD50), SAMN09296233 (RL50) to examine the interactions between $B B X$ genes, light-responsive genes, multiple hormone signaling genes, and anthocyanin biosynthesis genes in 'Rainier' fruits. The five PavBBX genes (PavBBX4, 6, 7, 9, and 11) were highly expressed during late fruit development and upregulated by light in 'Rainier' fruits. We analyzed the expression of nine anthocyanin biosynthesis genes (PAL, CHS, CHI, F3H, F3'H, DFR, ANS, UFGT, and MYB10) and 11 lightresponsive genes to identify genes that might regulate anthocyanin biosynthesis during fruit development. As shown in Figure 7, genes encoding light signaling components (HY5, PIF3) and anthocyanin biosynthesis genes were co-expressed with ABA, GA, and BR signaling pathway genes, suggesting that these three hormones might play major roles in light-dependent anthocyanin biosynthesis in sweet cherry fruits. Furthermore, the expression patterns of five PavBBX genes (PavBBX4, 6, 7, 9, and 11) were correlated with the expression patterns of anthocyanin biosynthesis genes and light-responsive genes. Notably, $B B X 6$ and $B B X 9$ expression levels were highly correlated with anthocyanin biosynthesis and light-responsive gene expression, and these genes were co-expressed with genes encoding components of the ABA, BR, and GA signaling pathways. These results showed that $B B X$ 
genes likely play major roles in regulating anthocyanin biosynthesis by integrating the light, $\mathrm{ABA}, \mathrm{GA}$, and $\mathrm{BR}$ signaling pathways.

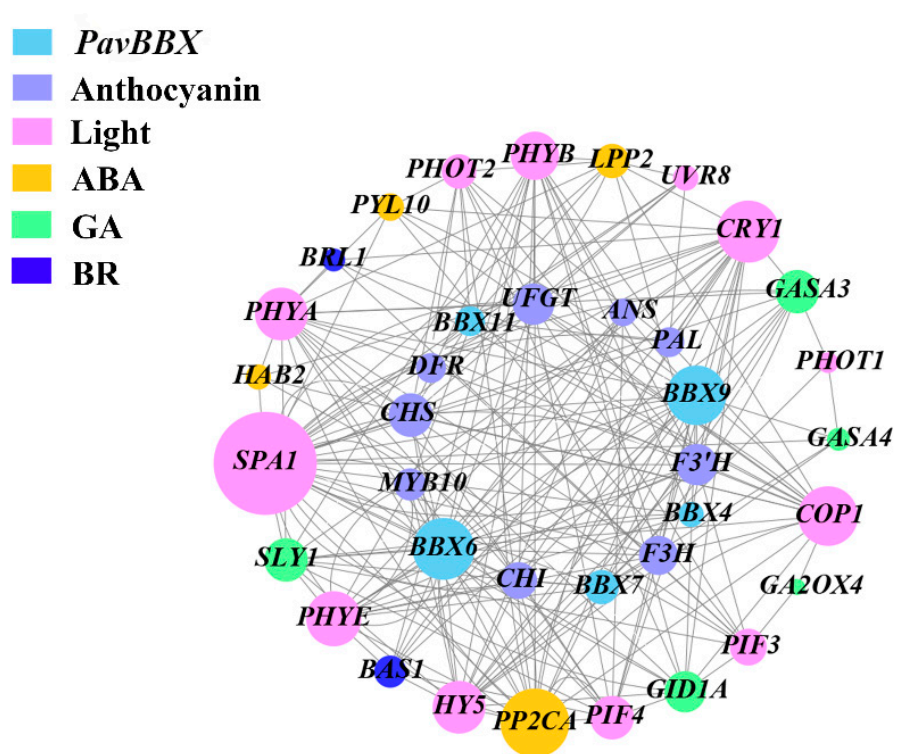

Figure 7. Co-expression network analysis of $B B X$, anthocyanin biosynthesis, light signaling, and multiple hormone signaling genes in bicolored cherry fruits.

\subsection{Regulation of PavBBX Family Gene Expression during Hormone Treatment}

Based on our analysis of changes in ABA, IAA, BR, MeJA, and $\mathrm{GA}_{3}$ contents in 'Rainier' fruits after light induction (Figure 6), we measured the expression levels of PavBBX genes in response to ABA, GA, and BR treatment by qRT-PCR (Figure 8). As expected, the PavBBX genes showed diverse expression patterns during treatment with different hormones. During ABA treatment, five PavBBX genes were significantly upregulated, indicating that these genes positively respond to $A B A$ treatment. A majority of PavBBX genes responded to GA, including PavBBX3, PavBBX4, PavBBX6, PavBBX8, PavBBX9, $P a v B B X 10$, and PavBBX14, which were significantly upregulated under GA treatment. The 15 PavBBX genes responded to BR to varying degrees, including PavBBX1 and PavBBX9, which were upregulated 4- to 5-fold compared to the control. Furthermore, PavBBX6 and $P a v B B X 9$ were significantly upregulated after treatment with the three hormones. In general, most PavBBX genes were sensitive to different hormone treatments. 

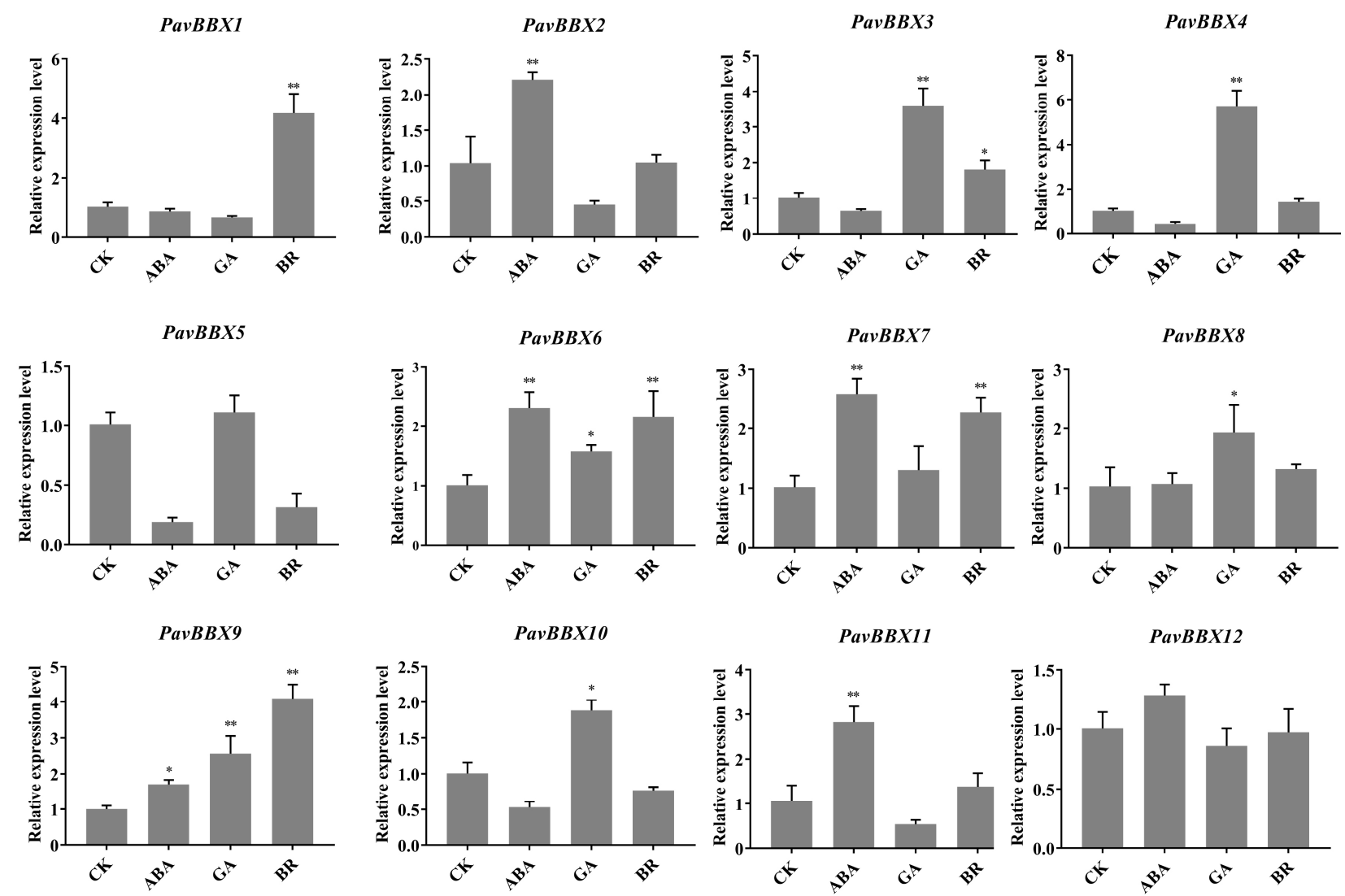

PavBBX13
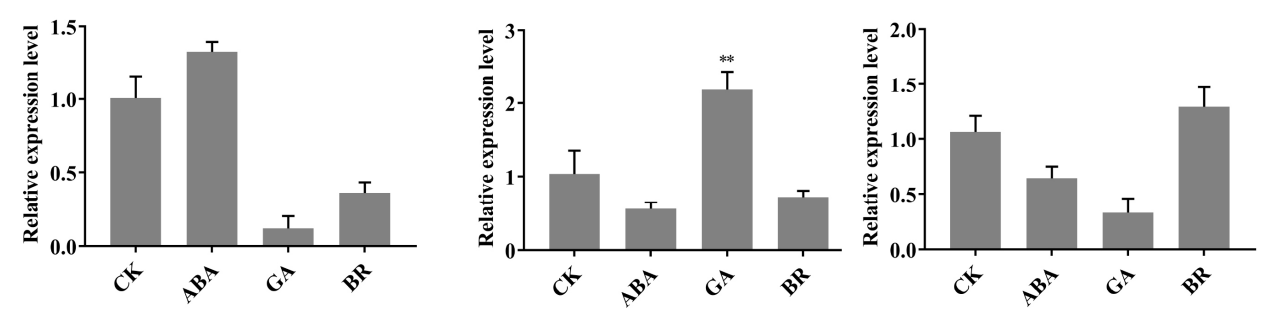

Figure 8. Expression profiles of sweet cherry $B B X$ genes in response to ABA, GA, and BR treatment. PavActin was used as the internal reference control to normalize template levels. Relative mRNA levels are represented as the mean $\pm S D(n=3)$. Statistically significant differences were assessed using Student's $t$-test $\left({ }^{*} p<0.05,{ }^{* *} p<0.01\right)$.

\subsection{Subcellular Localization of Sweet Cherry BBX Proteins}

Transcription factors play many regulatory roles in plants. The nuclear localization of transcription factors is important for their regulatory roles. Most $B B X$ proteins are located in the nucleus, such as $A t B B X 21, A t B B X 22[3,14]$. To examine the subcellular locations of $P a v B B X$ proteins in sweet cherry, we transiently transformed Nicotiana benthamiana epidermal cells with five PavBBX genes (PavBBX4, 6, 7, 9, and 11) and examined the subcellular localization of the resulting GFP-tagged fusion proteins. As shown in Figure 9, PavBBX4-GFP, PavBBX6-GFP, PavBBX7-GFP, PavBBX9-GFP, and PavBBX11-GFP showed green fluorescent signals in the nuclei of $N$. benthamiana epidermal cells. These results revealed that PavBBX4, PavBBX6, PavBBX7, PavBBX9, and PavBBX11 are nuclear proteins, which is consistent with previous results and their presumed roles as transcription factors $[3,14]$. 


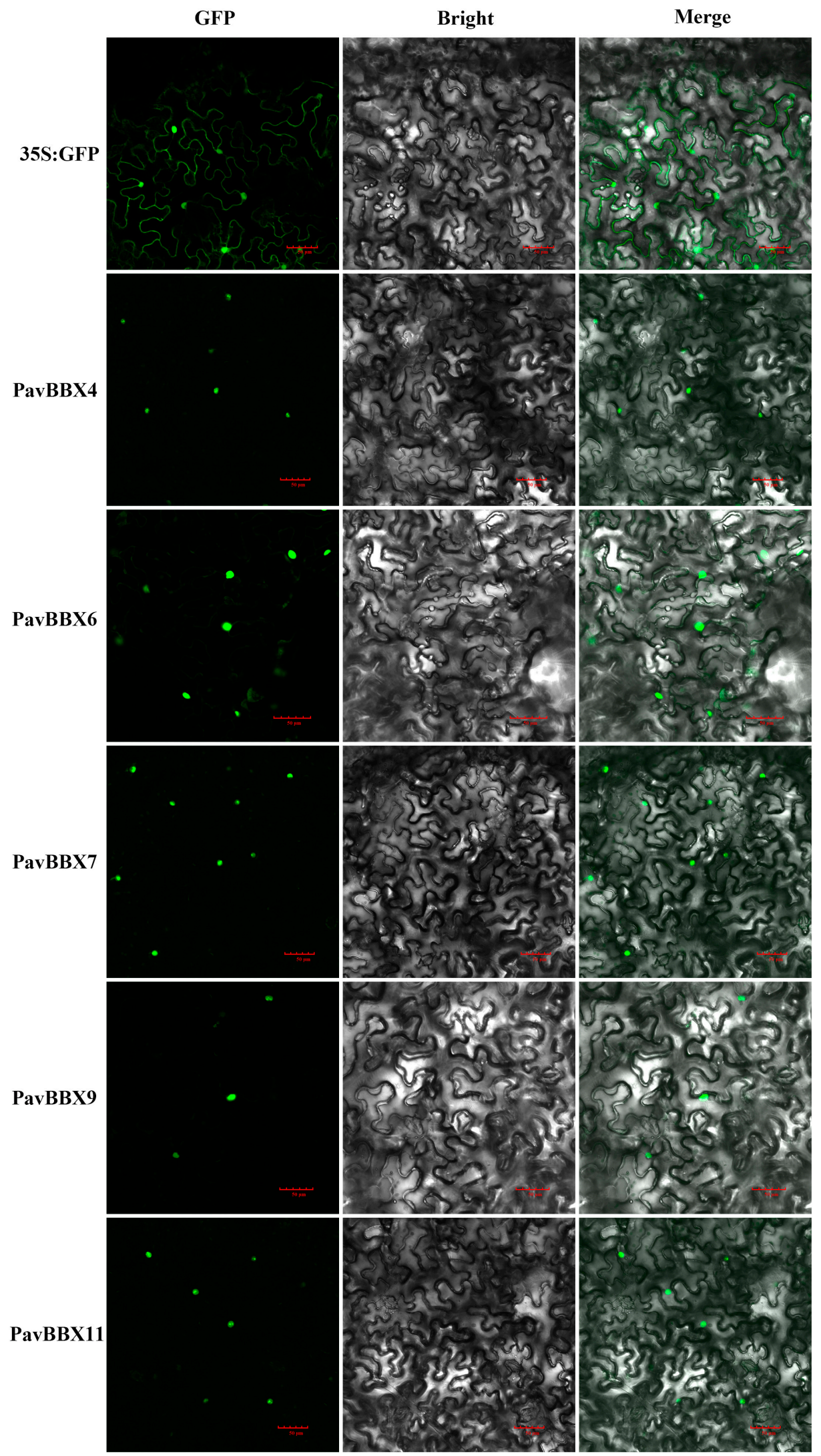

Figure 9. Subcellular localization of five GFP-fused PavBBX proteins. The five PavBBX-GFP fusion proteins (PavBBX4-GFP, PavBBX6-GFP, PavBBX7-GFP, PavBBX9-GFP, and PavBBX11-GFP) were transiently expressed in $N$. benthamiana leaves and observed by fluorescence microscopy $48 \mathrm{~h}$ later. Bar $=50 \mu \mathrm{m}$. 


\section{Discussion}

\subsection{Evolutionary Analysis of Sweet Cherry BBX Genes}

In the current study, we identified $15 B B X$ genes in the genome database of sweet cherry. The number of $B B X$ genes varies among plant species; for example, there are 32 $B B X$ family members in Arabidopsis [5], 30 in rice [41], 64 in apple [42], 25 in pear [37], and 29 in tomato [38]. These differences may be due to the differences in genome size and complexity among these species.

The PavBBX genes were divided into five subgroups based on multiple clustering analysis of $B B X$ genes of different species [7]. Although the BBX genes in other species also fall into five subgroups, the number of genes in each subgroup differs among species. In Arabidopsis thaliana, 7, 8, 4, and 13 AtBBX genes contain one B-box domain, two B-box domains, one $B$-box domain and one CCT domain, and two $B$-box domains and one CCT domains, respectively, while the corresponding numbers in sweet cherry are $3,6,2$, and 4 , respectively. In addition, subgroup I and II members contain two $B$-box domains plus one CCT domains; subgroup III members contain one $B$-box domain and one CCT domain; subgroup IV members contain two $B$-box domains; and subgroup V members contain one $B$-box domain. It was difficult to classify subgroups in sweet cherry based on the presence of conserved domains (Figures 1 and 2). PavBBX5 and PavBBX15 have no CCT domains even though they were assigned to subfamilies I and II; perhaps the CCT domain was lost from these genes during the process of evolution. The CCT domains are conserved and the two $B$-box domains are highly homologous in the different $B B X$ genes (Figures S2 and S3), indicating that $B B X$ genes arose early in the evolution of land plants.

\subsection{The Expression Patterns of PavBBX Genes in Sweet Cherry Fruit Development and Ripening}

Fruit development and ripening in sweet cherry is a complex physiological and biochemical process that is influenced by various transcription factors and regulatory proteins [36,43]. Many studies have shown that $B B X$ genes are involved in anthocyanin biosynthesis and fruit ripening [14,28]. For example, AtHY5 positively regulates anthocyanin biosynthesis [44], and AtBBX22 interacts with AtHY5 to promote anthocyanin biosynthesis [14]. The apple $B$-box protein $M d B B X 37$ modulates anthocyanin biosynthesis in conjunction with $M d M Y B 1, M d M Y B 9$, and MdHY5 [28]. In grapevine, the expression of $V v B B X 22$ proposed its involvement in fruit development and hormone response [4]. By contrast, MdBBX54 indirectly inhibits the expression of MdMYB1 by interacting with MdHY5 to suppress anthocyanin biosynthesis in apple [45]. In the current study, we demonstrated that PavBBX9 (the homolog of AtBBX22 in Arabidopsis) was significantly upregulated during late fruit development. This is consistent with previous findings [14]. Additionally, PavBBX4, PavBBX6, PavBBX7, and PavBBX11 were upregulated during late fruit development, which revealed that these four genes are involved in the ripening process in sweet cherry. Finally, PavBBX1, PavBBX2, PavBBX3, and PavBBX12 were downregulated during late fruit development, which means that these genes encode negative regulators of fruit ripening in sweet cherry.

\subsection{The Expression Patterns of PavBBX Genes in Plant Responses to Light and Multiple Hormones}

Light is an important factor that influences plant growth and development [40]. BBX proteins are primarily involved in seedling photomorphogenesis, the photoperiodic regulation of flowering, shade avoidance, and thermomorphogenesis [7,46-48]. These proteins also participate in the regulation of anthocyanin biosynthesis in fruits in the light [29]. For example, in pear, $P p B B X 18$ and $P p B B X 21$ antagonistically regulate light-induced anthocyanin biosynthesis via a competitive association with PpHY5 [49], and PpBBX16 regulates light-induced anthocyanin biosynthesis [29]. Additionally, in tomato, SlBBX19, SlBBX20 and SlBBX26 are light- and SIRIN-regulated, playing a role in fruit development ripening [50]. Anthocyanin accumulation is highly dependent on light in bicolored 'Rainier' cherries [36]. Based on our present results, seven PavBBX genes are upregulated dur- 
ing light exposure, proposed that these differentially expressed genes might function in light-induced anthocyanin biosynthesis.

Exogenous hormone treatment can promote fruit ripening [51]. Although sweet cherry has been classified as a non-climacteric fruit, ABA promotes the ripening of sweet cherry fruits, and ABA content in sweet cherry fruit increases strongly at the end of the color turning period and during the early stage of ripening [43]. The roles of PavBBX proteins in hormone signaling pathways are currently unclear. Several reports document the roles of $B B X$ genes in hormonal pathways in other plants [39,52-54]. In Arabidopsis, AtBBX21 regulates the light-mediated $A B A$ signaling pathway by suppressing the transcriptional activation of AtABI5, leading to shorter hypocotyls [39]. AtBBX24 might be involved in ethylene or brassinosteroid signaling based on its role in seedling photomorphogenesis in Arabidopsis [52]. Based on previous studies, we measured the hormone contents in 'Rainier' fruits that were bagged during fruit development, followed by bag removal, and found that $\mathrm{ABA}, \mathrm{GA}_{3}$, and $\mathrm{BR}$ contents increased at 48 and $96 \mathrm{~h}$ after bag removal (Figure 6). Furthermore, the expression patterns of $B B X$ genes were highly correlated with those of anthocyanin biosynthesis genes, light-responsive genes, and ABA, BR, and GA signaling pathway genes (Figure 7). RT-PCR revealed that the PavBBX genes were responsive to numerous hormonal treatments (Figure 8). The four PavBBX genes were regulated by more than one hormone treatment, providing that these genes may be involved in the interactions of different hormone signals at the physiological level. In addition, PavBBX6 and PavBBX9 responded to three hormone signals and light treatment, indicating that these genes encode proteins that integrate light and hormone signals to regulate anthocyanin biosynthesis. Together, these findings demonstrated that these PavBBX proteins might be involved in the crosstalk among multiple hormone signaling pathways and light, functioning as transcriptional regulators to modulate fruit development and ripening.

\section{Materials and Methods}

\subsection{Plant Growth Conditions and Hormone Treatments}

Bicolored 'Rainier' sweet cherry trees were grown under standard field conditions at the Beijing Institute of Forestry and Pomology, Beijing Academy of Agriculture and Forestry Sciences, Beijing, China. Fruit samples were collected at three time points during the growing season, including the green fruit expanding stage (15 days after flowering; $\mathrm{DAF})$, veraison (40 DAF), and the ripe stage (50 DAF), and used to study the expression characteristics of PavBBX genes.

A fruit bagging experiment was conducted at $15 \mathrm{DAF}$. The bags were removed from sweet cherry fruits at 45 days after flowering (45 DAF). Samples were taken at 0, 6, 12, 24, 48 , and $96 \mathrm{~h}$ after bag removal. There were three replicates performed in the experiment, with 10 fruits per replicate. The fruits were immediately frozen in liquid nitrogen and stored at $-80^{\circ} \mathrm{C}$.

Young fruits at 36 DAF were surface sterilized with $75 \%$ alcohol for $1 \mathrm{~min}$, rinsed twice with sterile distilled water, treated with $5 \%$ sodium hypochlorite for $13 \mathrm{~min}$, and rinsed three times with sterile distilled water. The fruits were cut into small squares, placed on solid callus induction medium (MS medium $+2.0 \mathrm{mg} / \mathrm{L} 6 \mathrm{BA}$, and $1.5 \mathrm{mg} / \mathrm{L} 2,4-\mathrm{D}$ ), and cultured for two weeks in the dark at $24 \pm 2{ }^{\circ} \mathrm{C}$. To investigate the effects of ABA, $\mathrm{BR}$, and GA treatment, calli subcultured on standard medium for 15 days were treated with $100 \mu \mathrm{mol} / \mathrm{L}$ of each phytohormone and cultured in the light at $24 \pm 2{ }^{\circ} \mathrm{C}$ under a $16 \mathrm{~h} / 8 \mathrm{~h}$ light/dark cycle. After 5 days, all of the calli were immediately frozen in liquid nitrogen and stored at $-80^{\circ} \mathrm{C}$ for further analysis. All treatments were performed in three biological replicates.

\subsection{Quantification of Total Anthocyanin Content}

The total anthocyanin levels in cherries were measured as previously described [36]. The pigment was extracted with methanol containing $0.1 \% \mathrm{HCl}$ in the dark at $-20{ }^{\circ} \mathrm{C}$ overnight, centrifuged at $4{ }^{\circ} \mathrm{C}$ for $15 \mathrm{~min}$ at $8000 \mathrm{rpm}$, then the supernatant was obtained, 
and the content of total anthocyanins was determined by differential $\mathrm{pH}$ method, with the light absorbance of each sample measured at wavelengths of 510 and $700 \mathrm{~nm}$ using a UV-vis spectrophotometer (Shimadzu, Kyoto, Japan) in buffers at pH 1.0 and pH 4.5 , respectively. Finally, the total anthocyanin content was expressed as mg cyanidin3-o-rutinoside (CGE)/100 mg fresh weight (FW). All samples were performed in three replicates.

\subsection{Identification of BBX Genes in the Prunus avium Genome}

The deduced amino acid sequences of 32 BBX genes in Arabidopsis thaliana were used as query sequences for a BlastP search of the sweet cherry genome database (http: / / cherry.kazusa.or.jp/). The CDD database (https:/ / www.ncbi.nlm.nih.gov/Structure/ cdd/cdd.shtml) and the SMART database (http:/ / smart.embl-heidelberg.de) were used to analyze the domains of the candidate $B B X$ proteins. The molecular weights, isoelectric points (pIs), and grand average of hydropathicity (GRAVY) values of the PavBBX proteins were calculated using the ExPASy website (https://web.expasy.org/protparam/).

\subsection{Phylogenetic Analysis and Gene Structure Analysis}

Multiple sequence alignments of BBX proteins were analyzed using ClustalW in BioEdit (http:/ / bioedit.software.informer.com), and the phylogenetic tree was constructed with the neighbor-joining algorithm in MEGA 6.0 [55]. Bootstrap analysis was carried out with 1000 replicates. Domains were identified with the SMART (http:/ / smart.emblheidelberg.de) and Pfam (http:/ / pfam.xfam.org) programs. WebLogo (http:/ / weblogo. berkeley.edu/logo.cgi) was used to generate sequence logos of the conserved domains.

\subsection{Cis-Element Prediction in the BBX Gene Promoters}

The promoter sequences ( $2 \mathrm{~kb}$ upstream from ATG) were extracted from Prunus avium whole genome scaffolds data (version 1.0), and cis-elements in the promoters were predicted using the PlantCARE online program (http:/ / bioinformatics.psb.ugent.be/ webtools/plantcare/html/).

\section{6. qRT-PCR Analysis}

Total RNA was extracted from the samples using a Plant Total RNA Extraction kit (Huayueyang Biotechnology, Beijing, China). The integrity of the RNA was examined by $1.0 \%$ agarose gel electrophoresis, and cDNA was synthesized from the RNA using a Reverse Transcription kit (TaKaRa Biotechnology, Dalian, China). All primers used for qPCR are listed in Supplementary Table S1. qRT-PCR was performed using SYBR Premix Ex Taq (Kangwei Century Biotechnology, Beijing, China) with the Rotor-Gene Real-Time PCR System. RT-qPCR analyses were performed using the conditions recommended in MIQE guideline [56], we screened five potential reference genes. including PavCAC, PavPP2A, PavSRP19, PavACT1, and PavActin [36,57]. The detailed sequences are shown in Table S1, and we analyzed the variation between samples (PavCAC, <2.5 Cq, PavPP2A and PavSRP19, <2.0 Cq, PavACT1, and PavActin <1.5 Cq). PavACT1 and PavActin were used presenting small variation between samples, and whose expression levels were expected to be constant over development and treatment. All experiments were carried out with three biological replicates. The relative expression value of each gene was quantified using the $2^{-\Delta \Delta C t}$ method (relative to PavActin) [58].

\subsection{Measuring $A B A, I A A, M e J A, G A_{3}$, and $B R$ Contents}

The ABA, IAA, MeJA, GA 3 , and BR contents of the samples were measured using ELISA (enzyme-linked immunosorbent assay) as described by [59]. All measurements were performed in three biological replicates. 


\subsection{Co-Expression Network Construction and Visualization}

The differentially expressed genes in the anthocyanin biosynthesis pathway, light signaling pathway, and hormone signaling-related pathways were identified in 'Rainier' sweet cherry (Table S2). Pearson's correlation tests were performed with SPSS v25 software using the FPKM values of both SAMN09296232 (RD50) and SAMN09296233 (RL50) samples. Any two genes with an absolute Pearson correlation coefficient of $\geq 0.9$ and a $p$-value of $\leq 0.05$ were considered to be significantly co-expressed genes. The co-expression network was visualized using Cytoscape v3.5.1 software.

\subsection{Subcellular Localization Analysis}

To examine the subcellular locations of the PavBBX proteins, five full-length PavBBX open reading frames (ORFs) (for PavBBX4, 6, 7, 9, and 11) without the stop codon were amplified from cDNA from 'Rainer' fruit. Each amplification product was cloned into the pCAMBIA1302 vector with green fluorescence protein (GFP) label under the control of the CaMV35S promoter. The gene-specific primers are listed in Table S3.

Nicotiana benthamiana plants were grown in a plant growth chamber at $26^{\circ} \mathrm{C}$ under a 16-h light/8-h dark regimen until they were approximately $15 \mathrm{~cm}$ tall and infiltrated with Agrobacterium strain EHA105 harboring the constructs described above. Infiltration was performed as described by [60]. The agroinfiltrated leaves were photographed 2 days after infiltration. GFP fluorescence images were captured using an Olympus laser-scanning confocal microscope.

\section{Conclusions}

In this study, we identified 15 PavBBX genes in the genome database of sweet cherry and systematically studied their gene structures and expression patterns. Our results illustrated that the PavBBX genes play important roles in fruit development and ripening, as revealed by $B B X$ gene expression patterns and changes in hormone levels in the light, and especially by the correlation between the expression of several $B B X$ genes and that of lightresponsive genes, multiple hormones signaling genes, and anthocyanin biosynthesis genes. Notably, the expression levels of several PavBBX genes increased in response to various hormone treatments including $\mathrm{ABA}, \mathrm{GA}$, and $\mathrm{BR}$, suggesting that these $B B X$ genes regulate anthocyanin biosynthesis by integrating light, ABA, GA, and BR signaling pathways. Taken together, our genome-wide analysis of the PavBBX family lays the foundation for further research on the biological functions of these genes in fruit development and ripening.

Supplementary Materials: Supplementary materials can be found at https: / www.mdpi.com/14 22-0067/22/4/1622/s1. Figure S1: Chromosome distribution of sweet cherry BBX genes. The scale on the left is in megabases (Mb). Figure S2: WebLogos showing the conserved domains in PavBBX proteins. A, B, and $C$ were obtained from protein sequence alignment of the $B$-box $1, B-b o x 2$, and CCT domains, respectively. The $x$-axis indicates the conserved sequences of the domain. The height of each letter indicates the conservation of each residue across all proteins. The y-axis shows a scale of the relative entropy, which reflects the conservation rate of each amino acid. Figure S3: Multiple sequence alignments of the conserved domains of the PavBBXs. Multiple sequence alignments of the $B$-box 1, B-box 2, and CCT domains are shown. The sequences were aligned using DNAMAN 6.0 and BioEdit. Table S1: Primer sequences used for RT-qPCR. Table S2: Abbreviations of differentially expressed genes. Table S3: Primer sequences used for gene amplification.

Author Contributions: Conceptualization, Y.W. and T.L. conceived and designed the experiments. Y.W., X.P., C.F. and Z.Z. performed the RNA extraction and gene expression analysis, Y.S., X.Z. (Xiang Zhang), Y.X., J.J., X.Z. (Xin Zhou) and W.W. performed subcellular localization and data analysis. Y.W. and T.L. wrote and revised the manuscript. All authors have read and agreed to the published version of the manuscript.

Funding: This research was funded by National Natural Science Foundation of China, grant number "31672119 and 31972390", National Key R\&D Program of China (2019YFD1000102-02) and 
Construction of Beijing Science and Technology Innovation and Service Capacity in Top Subjects (CEFF-PXM2019_014207_000032).

Institutional Review Board Statement: Not applicable.

Informed Consent Statement: Not applicable.

Data Availability Statement: The Arabidopsis, pear and tomato $B B X$ protein sequences were downloaded from the Arabidopsis information source (TAIR) database (http:/ / www.arabidopsis. org), GigaDB database (http:/ / gigadb.org/site/index) and Solanaceae genomics network (https: //solgenomics.net/). The sweet cherry RNA-seq data in response to light were retrieved from NCBI database (SRA accession numbers: SRP149590 (https:/ / www.ncbi.nlm.nih.gov/sra/SRP149590), respectively.

Acknowledgments: We are grateful to Kaichun Zhang (Beijing Institute of Forestry and Pomology, Beijing Academy of Agricultural and Forestry Sciences, Beijing, China) for the material support, Bingbing Li (China Agricultural University, China) for her helpful suggestions on the manuscript, and Kathleen Farquharson for providing language editing assistance.

Conflicts of Interest: The authors declare no conflict of interest.

$\begin{array}{ll}\text { Abbreviations } \\ \text { BBX } & \text { B-box } \\ \text { ABA } & \text { Abscisic acid } \\ \text { MeJA } & \text { Methyl jasmonate } \\ \text { BR } & \text { Brassinolide } \\ \text { GA } & \text { Gibberellic acid } \\ \text { IAA } & \text { Indole-3-acetic acid } \\ \text { CCT } & \text { CO, CO-like, TOC1 } \\ \text { DAF } & \text { Days after flowering } \\ \text { AA } & \text { amino acid residues } \\ \text { MW } & \text { molecular weight } \\ \text { pI } & \text { theoretical isoelectric point } \\ \text { GRAVY } & \text { grand average of hydropathicity } \\ \text { MS } & \text { Murashige and Skoog Medium } \\ \text { 6BA } & \text { N-(Phenylmethyl)-9H-purin-6-amine } \\ \text { 2,4-D } & \text { 2,4-Dichlorophenoxyacetic acid } \\ \text { ELISA } & \text { Enzyme-linked immunosorbent assay } \\ \text { FPKM } & \text { Fragments per kilobase of exon per million mapped fragments } \\ \text { ORF } & \text { Open reading frame } \\ \text { GFP } & \text { Green fluorescent protein } \\ \text { MIQE } & \text { Minimum information stablished for qRT-PCR experiments }\end{array}$

\section{References}

1. Riechmann, J.L.; Heard, J.; Martin, G.; Reuber, L.; Jiang, C.Z.; Keddie, J.; Adam, L.; Pineda, O.; Ratcliffe, O.J.; Samaha, R.R.; et al. Arabidopsis transcription factors: Genome-wide comparative analysis among eukaryotes. Science 2000, 290, $2105-2110$. [CrossRef]

2. Takatsuji, H. Zinc-finger transcription factors in plants. Cell Mol. Life Sci. 1998, 54, 582-596. [CrossRef]

3. Xu, D.Q.; Jiang, Y.; Li, J.G.; Lin, F.; Holm, M.; Deng, X.W. BBX21, an Arabidopsis B-box protein, directly activates HY5 and is targeted by COP1 for 26S proteasome-mediated degradation. Proc. Natl. Acad. Sci. USA 2016, 113, 7655-7660. [CrossRef]

4. Wei, H.R.; Wang, P.P.; Chen, J.Q.; Li, C.J.; Wang, Y.Z.; Yuan, Y.B.; Fang, J.G.; Leng, X.P. Genome-wide identification and analysis of B-BOX gene family in grapevine reveal its potential functions in berry development. BMC Plant Biol. 2020, 20, 72. [CrossRef] [PubMed]

5. Khanna, R.; Kronmiller, B.; Maszle, D.R.; Coupland, G.; Holm, M.; Mizuno, T.; Wu, S.H. The Arabidopsis B-box zinc finger family. Plant Cell 2009, 21, 3416-3420. [CrossRef] [PubMed]

6. Griffiths, S.; Dunford, R.P.; Coupland, G.; Laurie, D.A. The evolution of constans-like gene families in barley, rice, and Arabidopsis. Plant Physiol. 2003, 131, 1855-1867. [CrossRef]

7. Gangappa, S.N.; Botto, J.F. The BBX family of plant transcription factors. Trends Plant Sci. 2014, 19, 460-470. [CrossRef]

8. Putterill, J.; Robson, F.; Lee, K.; Simon, R.; Coupland, G. The constans gene of Arabidopsis promotes flowering and encodes a protein showing similarities to zinc-finger transcription factors. Cell 1995, 80, 847-857. [CrossRef] 
9. Robson, F.; Costa, M.M.R.; Hepworth, S.R.; Vizir, I.; Pineiro, M.; Reeves, P.H.; Putterill, J.; Coupland, G. Functional importance of conserved domains in the flowering-time gene constans demonstrated by analysis of mutant alleles and transgenic plants. Plant $J$. 2001, 28, 619-631. [CrossRef] [PubMed]

10. Yang, Y.J.; Ma, C.; Xu, Y.J.; Wei, Q.; Imtiaz, M.; Lan, H.B.; Gao, S.; Cheng, L.N.; Wang, M.Y.; Fei, Z.J.; et al. A zinc finger protein regulates flowering time and abiotic stress tolerance in chrysanthemum by modulating gibberellin biosynthesis. Plant Cell 2014, 26, 2038-2054. [CrossRef]

11. Datta, S.; Hettiarachchi, G.H.C.M.; Deng, X.W.; Holm, M. Arabidopsis constans-Like3 is a positive regulator of red light signaling and root growth. Plant Cell 2006, 18, 70-84. [CrossRef]

12. Cheng, X.F.; Wang, Z.Y. Overexpression of COL9, a CONSTANS-LIKE gene, delays flowering by reducing expression of CO and FT in A. thaliana. Plant J. 2005, 43, 758-768. [CrossRef]

13. Zhao, X.; Heng, Y.; Wang, X.; Deng, X.W.; Xu, D. A positive feedback loop of BBX11-BBX21-HY5 promotes photomorphogenic development in Arabidopsis. Plant Commun. 2020, 1, 100045. [CrossRef]

14. Chang, C.S.; Li, Y.H.; Chen, L.T.; Chen, W.C.; Hsieh, W.P.; Shin, J.; Jane, W.N.; Chou, S.J.; Choi, G.; Hu, J.M.; et al. LZF1, a HY5-regulated transcriptional factor, functions in Arabidopsis de-etiolation. Plant J. 2008, 54, 205-219. [CrossRef]

15. Zhang, X.; Huai, J.; Shang, F.; Xu, G.; Tang, W.; Jing, Y.; Lin, R. A PIF1/PIF3-HY5-BBX23 transcription factor cascade affects photomorphogenesis. Plant Physiol. 2017, 174, 2487-2500. [CrossRef]

16. Job, N.; Yadukrishnan, P.; Bursch, K.; Datta, S.; Johansson, H. Two B-box proteins regulate photomorphogenesis by oppositely modulating HY5 through their diverse C-terminal domains. Plant Physiol. 2018, 176, 2963-2976. [CrossRef] [PubMed]

17. Gangappa, S.N.; Holm, M.; Botto, J.F. Molecular interactions of BBX24 and BBX25 with HYH, HY5 homolog, to modulate Arabidopsis seedling development. Plant Sign. Behav. 2013, 8, e25208. [CrossRef] [PubMed]

18. Gangappa, S.N.; Crocco, C.D.; Johansson, H.; Datta, S.; Hettiarachchi, C.; Holm, M.; Botto, J.F. The Arabidopsis B-box protein BBX25 interacts with HY5, negatively regulating BBX22 expression to suppress seedling photomorphogenesis. Plant Cell 2013, 25, 1243-1257. [CrossRef]

19. Lin, F.; Jiang, Y.; Li, J.; Yan, T.T.; Fan, L.M.; Liang, J.S.; Chen, Z.J.; Xu, D.Q.; Deng, X.W. B-box domain Protein28 negatively regulates photomorphogenesis by repressing the activity of transcription factor HY5 and undergoes COP1-mediated degradation. Plant Cell 2018, 30, 2006-2019. [CrossRef] [PubMed]

20. Holtan, H.E.; Bandong, S.; Marion, C.M.; Adam, L.; Tiwari, S.; Shen, Y.; Maloof, J.N.; Maszle, D.R.; Ohto, M.A.; Preuss, S.; et al. BBX32, an Arabidopsis B-Box protein, functions in light signaling by suppressing HY5-regulated gene expression and interacting with STH2/BBX21. Plant Physiol. 2011, 156, 2109-2123. [CrossRef] [PubMed]

21. Osterlund, M.T.; Hardtke, C.S.; Wei, N.; Deng, X.W. Targeted destabilization of HY5 during light-regulated development of Arabidopsis. Nature 2000, 405, 462-466. [CrossRef] [PubMed]

22. Heng, Y.; Lin, F.; Jiang, Y.; Ding, M.; Yan, T.; Lan, H.; Zhou, H.; Zhao, X.; Xu, D.; Deng, X.W. B-Box Containing Proteins BBX30 and BBX31, acting downstream of HY5, negatively regulate photomorphogenesis in Arabidopsis. Plant Physiol. 2019, 180, 497-508. [CrossRef]

23. Yadav, A.; Bakshi, S.; Yadukrishnan, P.; Lingwan, M.; Dolde, U.; Wenkel, S.; Masakapalli, S.K.; Datta, S. The B-box-containing microprotein miP1a/BBX31 regulates photomorphogenesis and UV-B protection. Plant Physiol. 2019, 179, 1876-1892. [CrossRef] [PubMed]

24. Song, Z.; Bian, Y.; Liu, J.; Sun, Y.; Xu, D. B-box proteins: Pivotal players in light-mediated development in plants. J. Integr. Plant Biol. 2020, 62, 1293-1309. [CrossRef] [PubMed]

25. Song, Z.; Yan, T.; Liu, J.; Bian, Y.; Heng, Y.; Lin, F.; Jiang, Y.; Wang Deng, X.; Xu, D. BBX28/BBX29, HY5 and BBX30/31 form a feedback loop to fine-tune photomorphogenic development. Plant J. 2020. [CrossRef] [PubMed]

26. Nagaoka, S.; Takano, T. Salt tolerance-related protein STO binds to a Myb transcription factor homologue and confers salt tolerance in Arabidopsis. J. Exp. Bot. 2003, 54, 2231-2237. [CrossRef] [PubMed]

27. Fang, H.; Dong, Y.; Yue, X.; Hu, J.; Jiang, S.; Xu, H.; Wang, Y.; Su, M.; Zhang, J.; Zhang, Z.; et al. The B-box zinc finger protein MdBBX20 integrates anthocyanin accumulation in response to ultraviolet radiation and low temperature. Plant Cell Environ. 2019, 42, 2090-2104. [CrossRef]

28. An, J.P.; Wang, X.F.; Espley, R.V.; Lin-Wang, K.; Bi, S.Q.; You, C.X.; Hao, Y.J. An apple B-Box protein MdBBX37 modulates anthocyanin biosynthesis and hypocotyl elongation synergistically with MdMYBs and MdHY5. Plant Cell Physiol. 2020, 61, 130-143. [CrossRef]

29. Bai, S.; Tao, R.; Tang, Y.; Yin, L.; Ma, Y.; Ni, J.; Yan, X.; Yang, Q.; Wu, Z.; Zeng, Y.; et al. BBX16, a B-box protein, positively regulates light-induced anthocyanin accumulation by activating MYB10 in red pear. Plant Biotechnol. J. 2019, 17, 1985-1997. [CrossRef]

30. Xu, Y.; Zhao, X.; Aiwaili, P.; Mu, X.; Zhao, M.; Zhao, J.; Cheng, L.; Ma, C.; Gao, J.; Hong, B. A zinc finger protein BBX19 interacts with ABF3 to affect drought tolerance negatively in chrysanthemum. Plant J. 2020, 103, 1783-1795. [CrossRef]

31. Min, J.H.; Chung, J.S.; Lee, K.H.; Kim, C.S. The constans-like 4 transcription factor, AtCOL4, positively regulates abiotic stress tolerance through an abscisic acid-dependent manner in Arabidopsis. J. Integr. Plant Biol. 2015, 57, 313-324. [CrossRef] [PubMed]

32. Wang, Q.M.; Zeng, J.X.; Deng, K.Q.; Tu, X.J.; Zhao, X.Y.; Tang, D.Y.; Liu, X.M. DBB1a, involved in gibberellin homeostasis, functions as a negative regulator of blue light-mediated hypocotyl elongation in Arabidopsis. Planta 2011, 233, 13-23. [CrossRef] [PubMed] 
33. Fan, X.Y.; Sun, Y.; Cao, D.M.; Bai, M.Y.; Luo, X.M.; Yang, H.J.; Wei, C.Q.; Zhu, S.W.; Sun, Y.; Chong, K.; et al. BZS1, a B-box protein, promotes photomorphogenesis downstream of both brassinosteroid and light signaling pathways. Mol. Plant 2012, 5, 591-600. [CrossRef]

34. Zhang, H.; Zhang, Q.; Zhai, H.; Gao, S.P.; Yang, L.; Wang, Z.; Xu, Y.T.; Huo, J.X.; Ren, Z.T.; Zhao, N.; et al. IbBBX24 promotes the jasmonic acid pathway and enhances fusarium wilt resistance in sweet potato. Plant Cell 2020, 32, 1102-1123. [CrossRef]

35. Esti, M.; Cinquanta, L.; Sinesio, F.; Moneta, E.; Di Matteo, M. Physicochemical and sensory fruit characteristics of two sweet cherry cultivars after cool storage. Food Chem. 2002, 76, 399-405. [CrossRef]

36. Guo, X.; Wang, Y.T.; Zhai, Z.F.; Huang, T.J.; Zhao, D.; Peng, X.; Feng, C.; Xiao, Y.H.; Li, T.H. Transcriptomic analysis of light-dependent anthocyanin accumulation in bicolored cherry fruits. Plant Physiol. Bioch. 2018, 130, 663-677. [CrossRef]

37. Chu, Z.N.; Wang, X.; Li, Y.; Yu, H.Y.; Li, J.H.; Lu, Y.G.; Li, H.X.; Ouyang, B. Genomic organization, phylogenetic and expression analysis of the B-BOX gene family in tomato. Front. Plant Sci. 2016, 7, 1552. [CrossRef]

38. Cao, Y.P.; Han, Y.H.; Meng, D.D.; Li, D.H.; Jiao, C.Y.; Jin, Q.; Lin, Y.; Cai, Y.P. B-box genes: Genome-wide identification, evolution and their contribution to pollen growth in pear (Pyrus bretschneideri Rehd.). BMC Plant Biol. 2017, 17, 156. [CrossRef]

39. Xu, D.Q.; Li, J.G.; Gangappa, S.N.; Hettiarachchi, C.; Lin, F.; Andersson, M.X.; Jiang, Y.; Deng, X.W.; Holm, M. Convergence of light and ABA signaling on the ABI5 promoter. Plos Genet. 2014, 10, e1004197. [CrossRef]

40. Jaakola, L. New insights into the regulation of anthocyanin biosynthesis in fruits. Trends Plant Sci. 2013, 18, 477-483. [CrossRef]

41. Huang, J.Y.; Zhao, X.B.; Weng, X.Y.; Wang, L.; Xie, W.B. The rice B-Box zinc finger gene family: Genomic identification, characterization, expression profiling and diurnal analysis. PLoS ONE 2012, 7, e48242. [CrossRef]

42. Liu, X.; Li, R.; Dai, Y.Q.; Chen, X.S.; Wang, X.Y. Genome-wide identification and expression analysis of the B-box gene family in the apple (Malus domestica Borkh.) genome. Mol. Genet. Genomics 2018, 293, 303-315. [CrossRef]

43. Shen, X.J.; Zhao, K.; Liu, L.L.; Zhang, K.C.; Yuan, H.Z.; Liao, X.; Wang, Q.; Guo, X.W.; Li, F.; Li, T.H. A role for PacMYBA in ABA-regulated anthocyanin biosynthesis in red-colored sweet cherry cv. Hong Deng (Prunus avium L.). Plant Cell Physiol. 2014, 55, 862-880. [CrossRef]

44. Shin, D.H.; Choi, M.; Kim, K.; Bang, G.; Cho, M.; Choi, S.B.; Choi, G.; Park, Y.I. HY5 regulates anthocyanin biosynthesis by inducing the transcriptional activation of the MYB75/PAP1 transcription factor in Arabidopsis. Febs. Lett. 2013, 587, 1543-1547. [CrossRef]

45. Fang, H.C.; Dong, Y.H.; Yue, X.X.; Chen, X.L.; He, N.B.; Hu, J.F.; Jiang, S.H.; Xu, H.F.; Wang, Y.C.; Su, M.; et al. MdCOL4 interaction mediates crosstalk between UV-B and high temperature to control fruit coloration in Apple. Plant Cell Physiol. 2019, 60, 1055-1066. [CrossRef]

46. Kim, D.H.; Park, S.; Lee, J.Y.; Ha, S.H.; Lee, J.G.; Lim, S.H. A rice B-box protein, OsBBX14, finely regulates anthocyanin biosynthesis in rice. Int. J. Mol. Sci. 2018, 19, 2190. [CrossRef]

47. Yamawaki, S.; Yamashino, T.; Nakamichi, N.; Nakanishi, H.; Mizuno, T. Light-responsive double B-box containing transcription factors are conserved in physcomitrella patens. Biosci. Biotech. Bioch. 2011, 75, 2037-2041. [CrossRef]

48. Yeh, C.M.; Kobayashi, K.; Fujii, S.; Fukaki, H.; Mitsuda, N.; Ohme-Takagi, M. Blue light regulates phosphate deficiency-dependent primary root growth inhibition in Arabidopsis. Front. Plant. Sci. 2020, 10. [CrossRef]

49. Bai, S.L.; Tao, R.Y.; Yin, L.; Ni, J.B.; Yang, Q.S.; Yan, X.H.; Yang, F.P.; Guo, X.P.; Li, H.X.; Teng, Y.W. Two B-box proteins, PpBBX18 and PpBBX21, antagonistically regulate anthocyanin biosynthesis via competitive association with Pyrus pyrifolia Elongated hypocotyl 5 in the peel of pear fruit. Plant J. 2019, 100, 1208-1223. [CrossRef]

50. Lira, B.S.; Oliveira, M.J.; Shiose, L.; Wu, R.T.A.; Rosado, D.; Lupi, A.C.D.; Freschi, L.; Rossi, M. Light and ripening-regulated BBX protein-encoding genes in S. lycopersicum. Sci. Rep. 2020, 10, 19235. [CrossRef]

51. Forlani, S.; Masiero, S.; Mizzotti, C. Fruit ripening: The role of hormones, cell wall modifications, and their relationship with pathogens. J. Exp. Bot. 2019, 70, 2993-3006. [CrossRef]

52. Li, F.; Sun, J.J.; Wang, D.H.; Bai, S.N.; Clarke, A.K.; Holm, M. The B-Box family gene STO (BBX24) in A. thaliana. regulates flowering time in different pathways. PLoS ONE 2014, 9, e87544. [CrossRef]

53. Vaishak, K.P.; Yadukrishnan, P.; Bakshi, S.; Kushwaha, A.K.; Ramachandran, H.; Job, N.; Babu, D.; Datta, S. The B-box bridge between light and hormones in plants. J. Photoch. Photobio. B. 2019, 191, 164-174. [CrossRef]

54. Yadukrishnan, P.; Datta, S. Light and abscisic acid interplay in early seedling development. New Phytol. 2021, 229, 763-769. [CrossRef]

55. Tamura, K.; Stecher, G.; Peterson, D.; Filipski, A.; Kumar, S. MEGA6: Molecular evolutionary genetics analysis version 6.0. Mol. Biol. Evol. 2013, 30, 2725-2729. [CrossRef]

56. Bustin, S.A.; Benes, V.; Garson, J.A.; Hellemans, J.; Huggett, J.; Kubista, M.; Mueller, R.; Nolan, T.; Pfaffl, M.W.; Shipley, G.L.; et al. The MIQE guidelines: Minimum information for publication of quantitative real-time PCR experiments. Clin. Chem. 2009, 55, 611-622. [CrossRef]

57. Alkio, M.; Jonas, U.; Sprink, T.; van Nocker, S.; Knoche, M. Identification of putative candidate genes involved in cuticle formation in Prunus avium (sweet cherry) fruit. Ann. Bot. Lond. 2012, 110, 101-112. [CrossRef]

58. Livak, K.J.; Schmittgen, T.D. Analysis of relative gene expression data using real-time quantitative PCR and the $2^{-\triangle \triangle C t}$ method. Methods 2001, 25, 402-408. [CrossRef] 
59. Sun, L.A.; Zhang, M.; Ren, J.; Qi, J.X.; Zhang, G.J.; Leng, P. Reciprocity between abscisic acid and ethylene at the onset of berry ripening and after harvest. BMC Plant. Biol. 2010, 10, 257. [CrossRef]

60. Sparkes, I.A.; Runions, J.; Kearns, A.; Hawes, C. Rapid, transient expression of fluorescent fusion proteins in tobacco plants and generation of stably transformed plants. Nat. Protoc. 2006, 1, 2019-2025. [CrossRef] 